\title{
Physiological and Psychological Effects of Fluctuating Illumination on Heart Rate Variability in Concentration
}

\author{
Shun YAMAMOTO ${ }^{\dagger}$ and Takashi IRIKURA
}

\begin{abstract}
Shibaura Institute of Technology Graduate School of Engineering and Science, Department of Power and Energy, Faculty of Electrical Engineering and Computer Science
\end{abstract}

Received March 5, 2020, Accepted May 17, 2021

\begin{abstract}
Recently, fluctuation is attracting widespread interest in lighting field. However, little attention has been paid to the effects of fluctuation with HRV during tasks on autonomic nervous systems and doing concentrate by lightings. This study is aimed at the physical and psychological effects of fluctuating illumination on HRV during various tasks by task and ambient lightings. The results suggested that a certain types of fluctuating illumination on HRV derived in concentration tends to promote relaxation and does not tend to restrict sleepiness based on circadian rhythms.
\end{abstract}

KEYWORDS: fluctuating illumination, automatic nervous system, Heart Rate Variability, LF/HF

\section{Introduction}

Fluctuating illumination was received some attentions in decades ${ }^{1,2)}$, and recently has been getting more because of the development of LED dimming technology, which can be controlled to the millisecond ${ }^{3,4}$. Especially, fluctuating illumination has been used in the creation of relaxation space ${ }^{3,5}$. Fluctuating illumination is often designed to induce comfort.

Numerous studies have been conducted on the effect of fluctuating illumination on autonomic nervous system and relaxation. For instance, suppressions of eye fatigue and stress in mental task by 1 /f fluctuating illumination reflected sunshine filtering through foliage was demonstrated ${ }^{6}$. In addition, Komazaki et al. reported that fluctuating illumination imitated by Heart Rate Variability (HRV) could increase alpha waves and promote relaxation ${ }^{5}$. On the other hand, Ogawa et al. reported that sounds based on HRV derived from a participant who carried out physical tasks could stimulate sympathetic nerve activity ${ }^{7}$. However, few studies have investigated the effect of fluctuating illumination on HRV derived in concentration.

With this background, the purpose of the present study was to clarify the physical and psychological effects of fluctuating illumination on HRV in concentration.

\section{Method}

In the present study, preliminary and secondary experiments were conducted. In the preliminary experiment, HRV was investigated during various tasks, and fluctuating illumination patterns were obtained and based on these samples. In the secondary experiment, the effects of various fluctuating illumination conditions were investigated.

\subsection{Preliminary experiment}

The setup for the preliminary experiment was a general office space by task and ambient lighting, as shown in Figure 1. The ambient lighting consisted of fluorescent lamps. The task lighting consisted of a LED lamp with pulse-width modulation (PWM) control to change the desk illuminance accordingly for the tasks or for rest. The distribution of illuminance on the desk

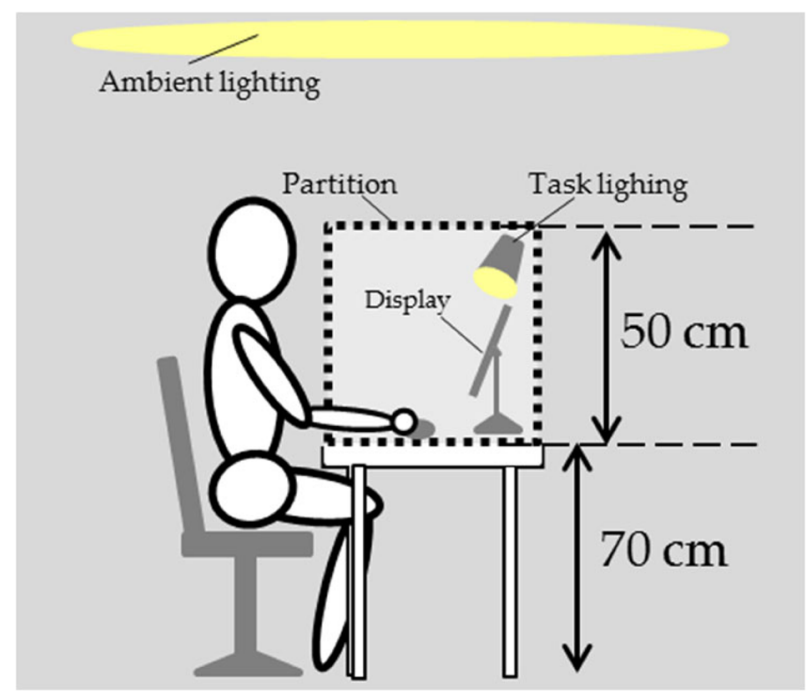

Figure 1 Experimental setup.

${ }^{\dagger}$ Corresponding Author: Shun Yamamotoｍa19089@shibaura-it.ac.jp 
Table 1 Preliminary experimental conditions.

\begin{tabular}{ll}
\hline Participants & $\begin{array}{l}\text { Four males in their 20s without color deficien- } \\
\text { cies }\end{array}$ \\
\hline Start time & $10: 00$ \\
\hline Desk illuminance & $\begin{array}{l}300 \mathrm{~lx} \text { (rest, minimum) } \\
600 \mathrm{~lx} \text { (task, minimum) }\end{array}$ \\
\hline Color temperature & $4500 \mathrm{~K}$ \\
\hline \multirow{3}{*}{ Tasks } & Krapelin test \\
& Sudoku \\
& Code matching/Cognitive judgment \\
\hline & Electrocardiogram \\
R-R interval in HRV \\
LF/HF \\
Critical Fusion Frequency (CFF)
\end{tabular}

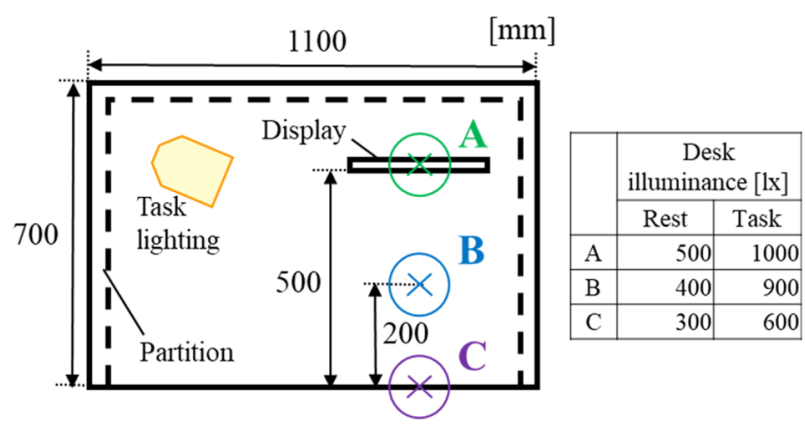

Figure 2 Distribution of desk illuminance.

is shown in Figure 2. Desk illuminance was enough for visual task on desk. In order to affect more by task lighting than by the display during Visual Display Terminals (VDT) tasks, the illuminance directly under the display was higher than that near participants.

The experimental conditions are shown in Table 1. Krapelin test is simple mental arithmetic with one digit on paper. Code matching task is to fill the box below numbers with each code corresponding each number written sample table on paper. Cognitive judgment is the task to judge correct combinations of fruits name, color of letters of these name and fruits picture, popping once per second on display. Code matching and cognitive task were done for each 5 min with 5 min rest between two tasks, and this cycle for $15 \mathrm{~min}$ was one type of tasks.

The experiment started at 10:00 when participants were the most active based on circadian rhythms.

At the beginning of the experiment, the participants who had confirmed and assented to the experiment were fitted with electrodes for monitoring electrocardiograms (ECGs) and asked to stay at rest for $10 \mathrm{~min}$. HRV at rest was then measured for $5 \mathrm{~min}$, followed by Critical Fusion Frequency (CFF) measurements. Next, the participants performed one of the mental tasks as above for $15 \mathrm{~min}$ while HRV was measured. CFF was then revaluated. The same procedure was continuity conducted for other two types of tasks after $10 \mathrm{~min}$ rest. The order of the tasks was changed for each participant, and each participant could practice each of the three types of tasks shown in Table 1.

After the experiments, the $\mathrm{R}$ wave to $\mathrm{R}$ wave interval (RRI) was determined for each participant's HRV, and RRI frequency analysis was carried out using an autoregressive model with past 16 data. Then, the total power spectrum in the low frequency (LF; 0.004$0.15 \mathrm{~Hz}$ ) bands and that in the high frequency (HF; $0.15-0.4 \mathrm{~Hz}$ ) bands were calculated. Then the LF/HF ratio $(\mathrm{LF} / \mathrm{HF})$, which indicates sympathetic nerve activity, was obtained.

\subsection{Secondary experiment}

The setup for the secondary experiment was the same as that for the preliminary experiment shown in Figure 1. The distribution of desk illuminance is shown in Figure 3.

In this experiment, only VDT task was conducted, and participants had been gazing only display during the VDT task. Thus, the desk illuminance near participants was set to less level than that in preliminary experiment.

The experimental conditions are shown in Table 2 . Vigilance task is to watch number popping once per second in a low on the display and to click if different odd numbers are popped successively three times. Since individual deference of work efficiency slightly affects work speed and stress, the task was selected.

Morningness-Eveningness Questionnaire (MEQ) ${ }^{8)}$ assesses life habits, and higher MEQ score indicates more morningness. MEQ consists 19 questions with each score about these life habits, for instance, getting up time, bedtime, suitable time for hard mental task or hard physical task, condition on each time, and so on. Participants answered these questions, and they were judged whether big shifts in life habit by MEQ score.

The Kwansei Gakuin Sleepiness Scale (KSS) ${ }^{9}$ valued subjective sleepiness by a feeling of activity shown in Table 3. In Table 3, there is the correspondence of feelings and KSS scores, which indicates higher KSS scores suggest higher subjective sleepiness. KSS score was conducted to answer how participants felt, showing these feelings in Table 3.

In general, alcohol and caffeine disturb sleepiness and autonomic nerve systems, and these disturbance affects circadian rhythm. On the other hands, the satiety center is stimulated by rising enough blood glucose level and satiety center activity causes feeling satiety and parasympathetic nerve activity ${ }^{10}$. In addition, while the stomach is full of foods, stomach acids are secreted by parasympathetic nerve actively, and the digestion in the stomach takes $2-4 \mathrm{~h}$ after eating. Therefore, partici- 


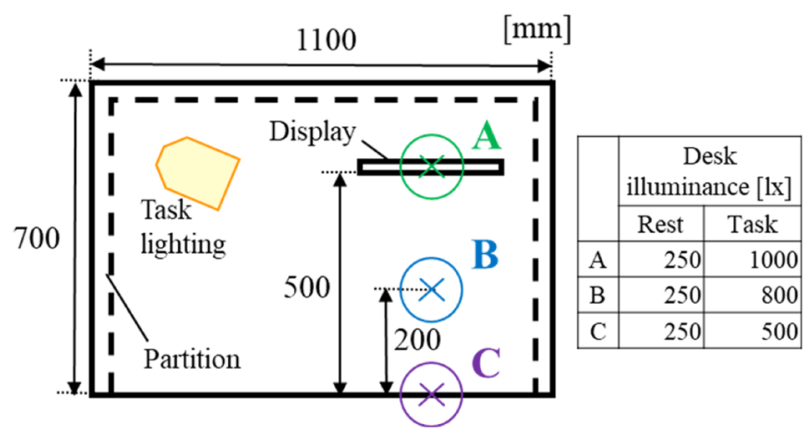

Figure 3 Distribution of desk illuminance.

Table 2 Secondary experimental conditions.

\begin{tabular}{ll}
\hline Participants & $\begin{array}{l}\text { Four males in their 20s without color deficien- } \\
\text { cies }\end{array}$ \\
\hline Start time & $10: 00$ \\
\hline Desk illuminance & $\begin{array}{l}3001 \mathrm{x} \text { (rest, minimum) } \\
6001 \mathrm{x} \text { (task, minimum) }\end{array}$ \\
\hline Color temperature & $4500 \mathrm{~K}$ \\
\hline \multirow{3}{*}{ Tasks } & Krapelin test \\
& Sudoku \\
& Code matching/Cognitive judgment \\
\hline \multirow{3}{*}{ Measurements } & Electrocardiogram \\
& R-R interval in HRV \\
& LF $/ H F$ \\
CFF
\end{tabular}

pants were instructed to get adequate sleep and refrain from consuming any alcohol or caffeine $24 \mathrm{~h}$ before the experiment. And participants were also instructed to avoiding overeating why they had felt satiety $2 \mathrm{~h}$ before the experiment, considering lunch time.

The Before the experiment, all participants were asked to complete the MEQ and practiced vigilance task because of avoiding the effect of proficiency.

Experiment started at 15:00 when sleepiness was stimulated in all participants based on circadian rhythms. The preparations, rest intervals, and measurements used in the secondary experiment were similar to those used in the preliminary experiment except for KSS. The participants performed vigilance tasks via a display for $15 \mathrm{~min}$ while ECGs were monitored under different lighting conditions mentioned later. After the task, CFF and KSS scores were evaluated. The correct answer rate and task response speeds were also recorded. The order of lighting conditions was changed for each participant to counterbalance any order effects.

The illuminance of the ambient lighting was fixed for all experiments, and fluctuating illumination was created using PWM control.

A fluctuation pattern (so call base fluctuation [BF]) was obtained from the 1/f fluctuation based on the RRI, which was derived from HRV in concentration at the
Table 3 KSS evaluation.

\begin{tabular}{lc}
\hline \multicolumn{1}{c}{ Feeling } & KSS score \\
\hline Full of vitality & 0.58 \\
Enough energy & 0.82 \\
Very efficient & 1.22 \\
Light steps & 1.56 \\
Broad outlook & 1.71 \\
No difficult to think & 2.11 \\
Slightly quick & 2.38 \\
Not languid & 3.03 \\
Comfortably and at home & 3.46 \\
No languid and no refreshed & 3.63 \\
Not careless & 3.95 \\
Easy to mistake & 4.21 \\
Some sleepiness without action & 4.39 \\
Not refreshed & 4.68 \\
Dull thinking & 4.86 \\
Dazed & 5.10 \\
Bleary eyes & 5.37 \\
Heavy eyelids & 5.54 \\
Long for bed & 5.74 \\
Fighting sleepiness & 6.17 \\
Shutting eyelids unconsciously & 6.33 \\
Break down by sleepiness & 6.49 \\
\hline & \\
\hline
\end{tabular}

preliminary experiment. Considering individual difference of RRI between participants, the ratio of the RRI average for each participant was multiplied by that of participants whose HRV was selected for the secondary experiment. Next, another fluctuation pattern, which was $\mathrm{BF}$ multiplied by 0.9 (so call $\mathrm{BF} \times 0.8$ ) on the time axis was prepared, since participants may be more affected by RRI derived in concentration with the difference from RRI in rest emphasized ${ }^{7}$. For comparisons, a fixed illumination pattern that had the same average illuminance of the two fluctuation patterns was used. Okiyama et al. reported the perceptive threshold of fluctuating illuminance on the white paper was $4-10 \%$ of reference illuminance without any tasks ${ }^{11)}$. Considering a decline in the perceptive by the vigilance tasks, the amplitude of both fluctuations was set to $8 \%$ of reference illuminance, $80 \mathrm{~lx}$ directly under the display.

These three patterns were selected as the illuminance conditions and created by the task lighting, as shown in Figure 1.

\section{Results and discussion}

\subsection{Preliminary experiment \\ 3.1.1 Declining rates of CFF}

The declining rates of CFF were calculated as shown in Eq. 1. The average declining rates for code matching and cognitive judgment, the Kraepelin test, and Sudoku were $1.60 \%, 2.10 \%$, and $2.80 \%$, respectively. No significant differences were found between tasks based on an analysis of variance (ANOVA) $(F(2,4)=1.685, p>1)$. 


$$
\begin{aligned}
& \text { Declining CFF rate } \\
& \quad=\frac{C F F(\text { before task })-C F F(\text { after task })}{C F F(\text { before task })} \times 100[\%]
\end{aligned}
$$

Okubo et al. reported that a greater than 5\% declining rate of CFF indicated some difficulties in mental tasks ${ }^{12}$. In this experiment, the largest average declining rate was that for Sudoku, but was still $<5 \%$. Thus, none of the tasks in this experiment caused substantial mental stress. In addition, there was little possibility that the participants feel over mental stress under fluctuating illumination with any HRV date in the experiments which had potential of reproducing mental in tasks.

\subsection{2 $\mathrm{LF} / \mathrm{HF}$}

The LF/HF averages obtained from the HRV analysis are shown in Figure 4. The bars in the figure represent standard errors. No significant differences were found on the ANOVA between before and after the tasks $(F(4,4)=0.5838, p>.1)$. As shown in Figure 4 , the average $\mathrm{LF} / \mathrm{HF}$ before the tasks was higher than the overall average.

This finding suggests that sympathetic nerve activ-

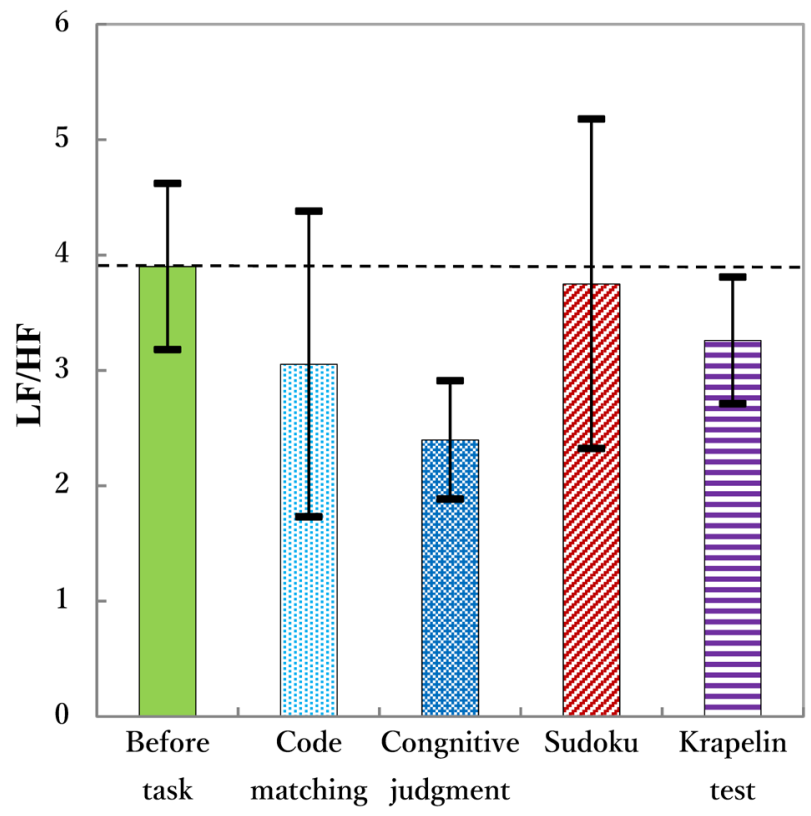

Figure 4 LF/HF averages.

Table 4 Electrocardiogram data from a selected participant.

\begin{tabular}{lccr}
\hline & $\begin{array}{c}\text { RRI } \\
\text { Average } \\
{[\mathrm{ms}]}\end{array}$ & $\begin{array}{c}\text { RRI } \\
\text { SD } \\
{[\mathrm{ms}]}\end{array}$ & LF/HF \\
\hline Before task & 750 & 55 & 4.57 \\
Krapelin & 636 & 45 & 4.17 \\
Code matching/Cognitive judgment & 693 & 48 & 2.57 \\
Sudoku & 678 & 42 & 6.99 \\
Sudoku between 10-15min & 659 & 42 & 10.26 \\
\hline
\end{tabular}

ity before the tasks was increasing; in other words, the participants were concentrating or straining more before the tasks. Considering this result and that regarding the declining $\mathrm{CFF}$, it appears that the participants did not need to concentrate much because these were simple tasks that did not require complex thinking. Otherwise, the participants must have felt stress before entering the room to carry out the tasks because they generally did not adapt to the room.

However, as shown in Table 4, the LF/HF in one participant who had been carrying out the Sudoku task between $10-15 \mathrm{~min}$ was remarkably higher than that of the other participants and that during other tasks, shown in Table 4. These result means this participant had been strongly concentrating during last $5 \mathrm{~min}$ of Sudoku. Therefore, the RRI variability series at this time was selected as the base for the fluctuation patterns in the secondary experiments.

\subsection{Secondary experiment}

\subsubsection{MEQ and KSS scores before the experiments}

The number of each life habits valued by MEQ are shown in Table 5 . These results suggested that almost participants have similar lifestyle and None of them has big shift in their life habits by MEQ.

It is considered that the KSS scores before the experiments were mainly affected by sleepiness derived from circadian rhythms, because analysis revealed a sig-

Table 5 The number of MEQ type.

\begin{tabular}{lccccc}
\hline Type & $\begin{array}{c}\text { Definitely } \\
\text { Evening }\end{array}$ & $\begin{array}{c}\text { Moderately } \\
\text { Evening }\end{array}$ & Middle & $\begin{array}{c}\text { Moderately } \\
\text { Morning }\end{array}$ & $\begin{array}{c}\text { Definitely } \\
\text { Morning }\end{array}$ \\
\hline Score & $16-30$ & $31-41$ & $42-58$ & $59-69$ & $70-86$ \\
Number & 0 & 3 & 6 & 1 & 0 \\
\hline
\end{tabular}

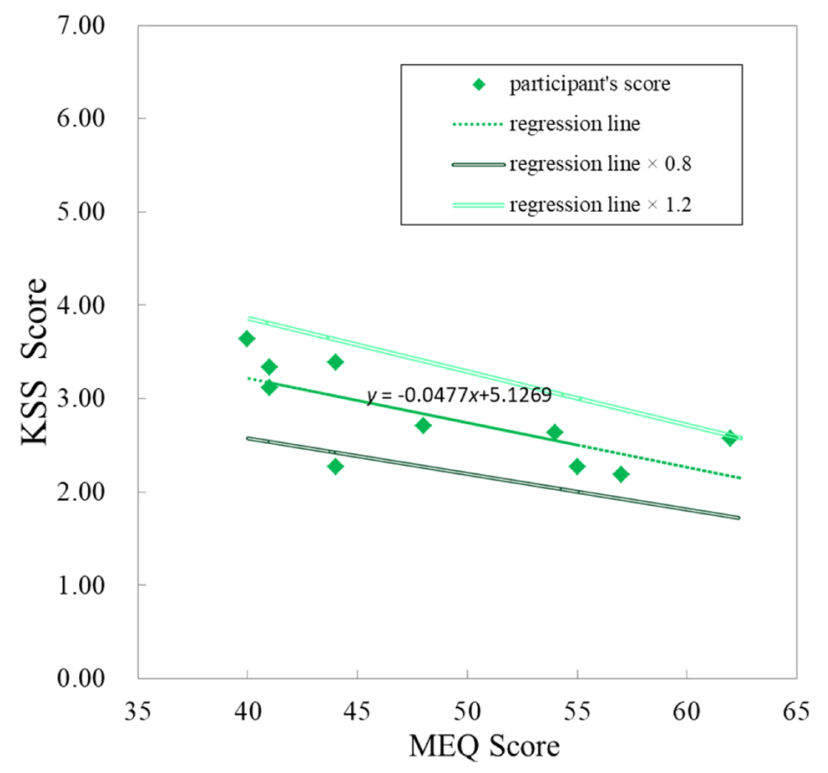

Figure 5 MEQ-KSS correlation. 
nificant negative correlation $(r=-0.7154, p<.01)$. Hence, a regression line was calculated and shown in Figure 5.

According to the relationship between the KSS and MEQ scores shown in Figure 5, almost all of the participants showed a small shift in circadian rhythms, although some individual differences were seen. Nevertheless, a certain participant whose KSS score before the experiments was 0.8 to 1.2 times over the regression line tended to have a big shift in circadian rhythms. Therefore, 1 participant was excluded from the analysis.

\subsubsection{Declining rates of CFF, correct rates, and task response speeds}

The average declining rates of CFF calculated using Eq. 1 are shown in Figure 6. The bars in the figure represent standard errors. An ANOVA revealed marginally significant differences; however, no significant differences were found between conditions, as the average declining rates were higher under both types of fluctuating than under fixed illumination in contrast to previous study ${ }^{6}$.

This result suggested that illumination changing in the range of $\pm 80 \mathrm{~lx}$ tended to cause more eye strain with about 3\% declining rate of CFF shown in Figure 6. Thus, there was little possibility that fluctuating illumination caused excessive eye strain because the declining rate was $<5 \%$ as above ${ }^{10)}$. However, the suitable range of fluctuating illumination may be narrow, considering eye strain and mental stress under fluctuating lightings in long-term.

No significant differences were seen in the correct answer rate or task response speeds, which suggest that fluctuating illumination does not interfere with working in short time. However, due to eye strain and mental stress as above, work efficiency slightly tends to be reduced under fluctuating lightings in long-term

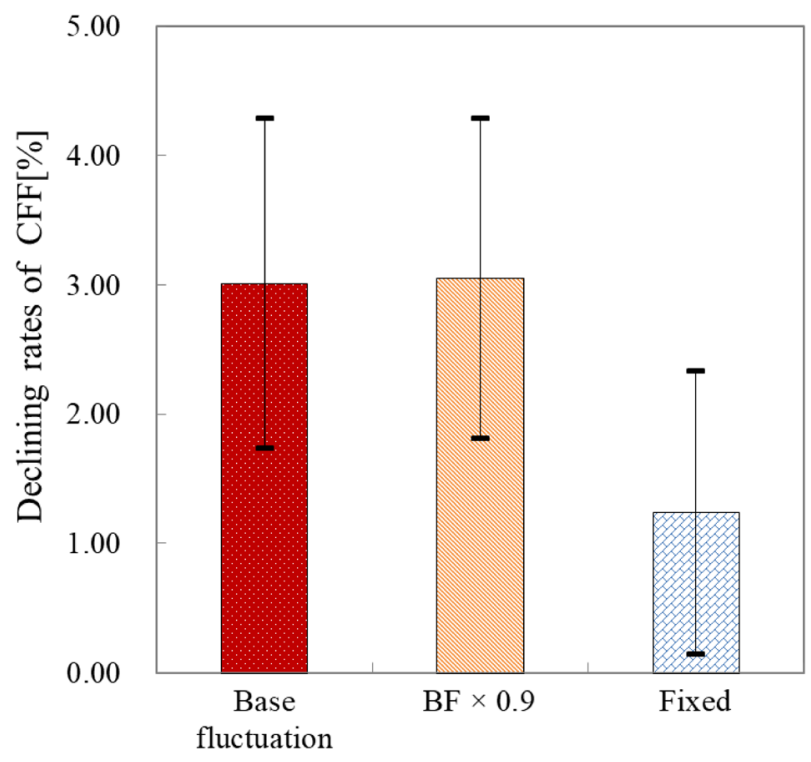

Figure 6 Declining rate of CFF. than decades min.

\subsection{3 $\mathrm{LF} / \mathrm{HF}$}

Average LF/HF values are shown in Figure 7. The bars in the figure represent standard errors. Based on an ANOVA, a marginally significant difference was found $(F(2,9)=2.5463, p<1)$. Although no significant differences were found on multiple regression analysis, $\mathrm{LF} / \mathrm{HF}$ under the fixed lighting condition was higher than that under both fluctuating conditions, which indicated rising sympathetic nerve activity. This result suggested that fluctuating illumination promotes relaxation by inhibiting the stimulation of sympathetic nerve activity.

However physiological effects of sound stimuli with HRV derived in concentration was contrary tendency,

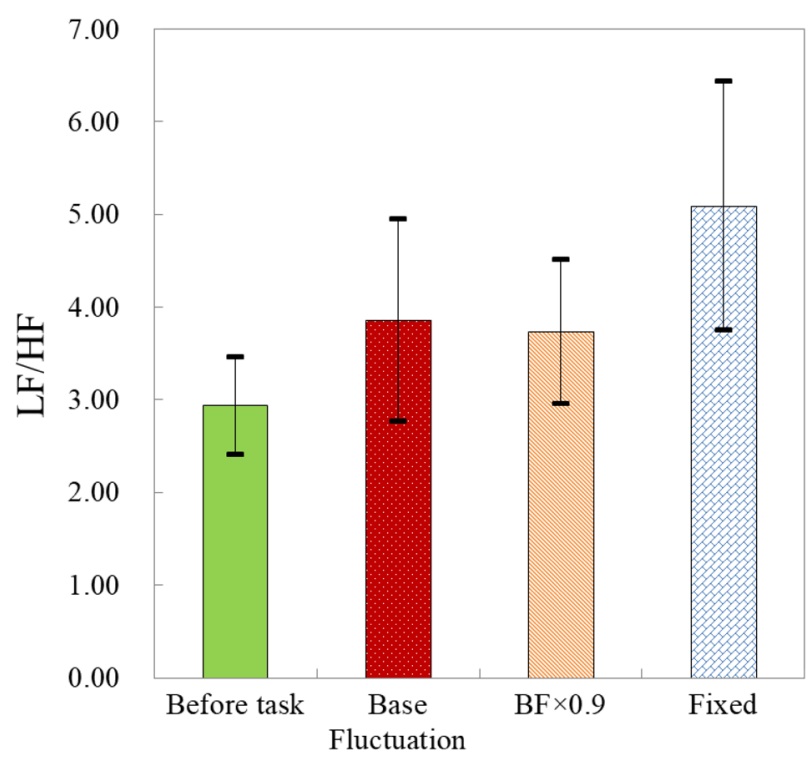

Figure 7 LF/HF averages.

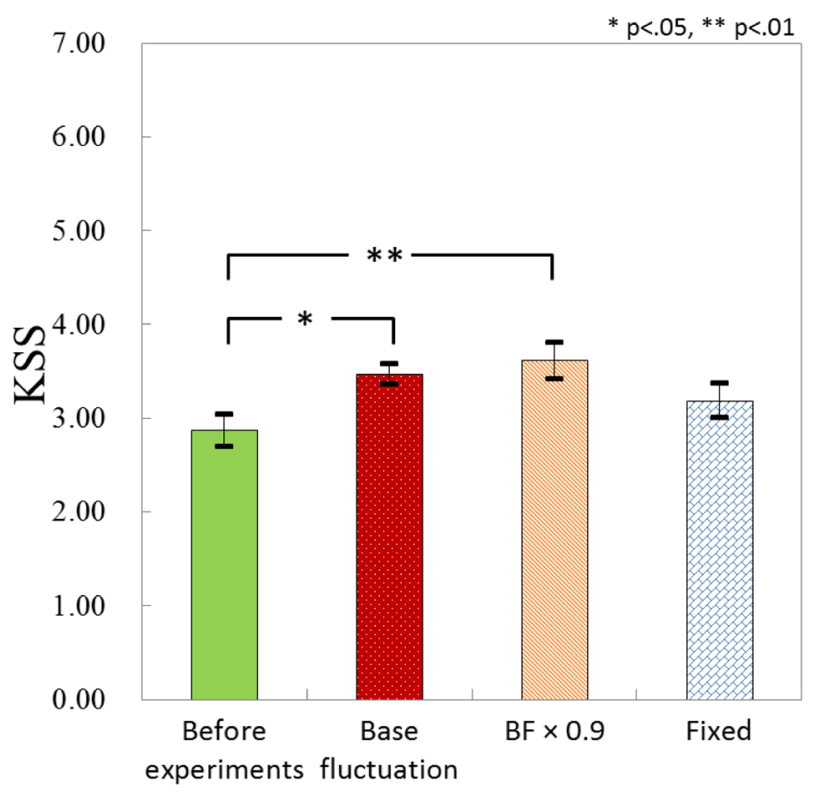

Figure 8 KSS scores averages. 
promoting activity ${ }^{7}$. These contrary results might be caused by differences of tasks for deriving HRV and fluctuation patterns, in addition to differences of stimuli. In previous study, physical tasks for deriving HRV was pedaling an exercise bicycle by between $42-87 \mathrm{rpm}$ with 20 Watt loads, which means more stress was caused. Furthermore, fluctuation patterns were obtained with tone burst wave like heartbeat. These differences have possible to do different physiological effects.

\subsubsection{Subjective sleepiness by KSS}

The average KSS scores are shown in Figure 8. An ANOV A revealed significant differences $(F(2,9)=7.1985$, $p<.01)$. The bars in the figure represent standard errors. Multiple regression analysis indicated significant increases in KSS scores under both base fluctuation $(p<.05)$ and $\mathrm{BF} \times 0.9(p<.01)$. This finding and KSS evaluation shown in Table 3 suggests that fluctuating illumination stimulates sleepiness more than fixed illumination but not extreme.

Generally, afternoon sleepiness is increased by circadian rhythms or parasympathetic nerve activity in having lunch at 14:00-16:00. Whereas sympathetic nerve activity promotes concentration and restricts sleepiness. In fact, fixed lighting did not inhibit the sympathetic nerve activity during tasks, and restrict sleepiness more than both fluctuation ones, as shown in Figures 7-8. These means that more sleepiness under both fluctuating conditions was brought about by circadian rhythms, because fluctuating illumination inhibited the stimulation of sympathetic nerve activity.

\section{Conclusion}

The results of the present study in regard to the performance of mental tasks and HRV under fluctuating illumination indicated the following:

(1) Short-term exposure to fluctuating illumination less than $15 \mathrm{~min}$ has slight risk of excessive eye strain and reduced work efficiency.

(2) Certain types of fluctuating illumination on HRV derived in concentration tend to promote relaxation.

(3) Fluctuating illumination on HRV derived in concentration does not tend to restrict sleepiness based on circadian rhythms.

\section{References}

(1) Seki, H., Tsukamoto, H. and Oguchi, K.: Experimental lighting setups with $1 / \mathrm{f}$ fluctuation controller, J. Illum. Eng. Inst. Jpn., 75 (Appendix), p. 152
(1992).

(2) Oguchi, K. and Tsukamoto, H.: Measurement of fluctuations in illuminance of natural and artificial light, Light. Res. Technol., 25-4, pp. 179-181 (1993).

(3) Nishide, R., Hashimoto, K., Sano K., Minami, K., Mitani, T., Nagai, Y. and Taura, T.: Trial manufacture of soft luminescence based of organic electroluminescence technology, Proceedings of the Annual Conference of JSSD, the 53rd Annual Conference of JSSD (2006).

(4) Fukuda, M.: A Driving Circuit for an LED with $1 / \mathrm{f}$ fluctuation-Lighting with healing-, Bulletin of Photonics Research Center, Chitose Institute of Science and Technology, 2-1, p. 23 (2003).

(5) Komazaki, M., Shinzawa, N., Nakamura, T., Konno, N., Shimada, T. and Miyaho, N.: Study on healing effect of $1 / \mathrm{f}$ characteristics of visible LED light to human beings, Electronics Society, pp. 36 (2008).

(6) Takahashi, H. and Irikura, T.: Effect of 1/F Fluctuation in Illumination on work, the 10th Asia Lighting Conference, pp. 319-321 (2017).

(7) Ogawa, K., Shibagaki, Y. and Hagiwara, H.: Effects of sound stimuli created from luctuations in heartbeat intervals on fluctuations in subjects' heartbeat intervals, Japan Ergonomic. Soc., 44-6, pp. 334-340 (2009).

(8) Ishihara, K., Miyashita, A., Inugami, M., Fukuda, K., Yamazaki, K. and Miyata, H.: The results of investigation by the Japanese version of Morningness Eveningness Questionnaire, Jpn. J. Phycol., 57-2, pp. 87-91 (1986).

(9) Ishihara, K., Saito, T. and Miyata, Y.: Sleepiness scale and an experimental approach, Jpn. Psychol. Res., 52-6, pp. 362-365 (1982).

(10) Hongo, M.: The stomach acids secretion, the secretion in stomach and the stomach motility, The chemistry and education, Chem. Soc. Japan, 65-7, pp. 356-357 (2017).

(11) Okiyama, K. and Okajima, K.: Illuminance modulation perception as a functions of temporal frequency, Proceeding of the 43th The Illuminating Engineering Institute of Japan National Convention, 8-7 (2010).

(12) Okubo, T. and Kato, Z:: The measurements of bio function for beginners, NIPPON.

Part of this work was presented at the 12th Asia lighting conference, August 23th, Daegu, Korea 